\title{
CORRESPONDENCE
}

\section{Scholars' awards go mainly to men}

One area in which female scientists still have to confront sexism (Nature 468,$733 ; 2010)$ is in scholarly awards: women win fewer. Scientific societies must examine practices for selecting awardees.

The proportion of women receiving service or teaching awards in the past two decades is roughly equivalent to the proportion of women within the cohort-adjusted $\mathrm{PhD}$ pool in that discipline, but only half of these have won scholarly awards. Using data in the public domain on 13 disciplinary societies, we found that the proportion of female prizewinners in ten of these was much lower than the proportion of female full professors in each discipline (see also P. Leboy The Scientist 22, 67; 2008).

Our investigations reveal that practices for selecting awardees all tend to operate with few guidelines, minimal oversight and little attention to conflict-ofinterest issues. Having women on selection committees helps recognition, but many panels have no female members and few have female chairs.

The pool of female nominees for an award is typically small. Their nomination letters tend to contain fewer descriptors of exceptionality, use stereotypically female adjectives (such as 'cooperative' and 'dependable') and mention personal details. Notices soliciting nominations, by contrast, tend to use language that fosters male images, such as 'decisive' or 'confident'. Unsurprisingly, bias thrives under these conditions.

To help push through key changes, the Association for Women in Science in Virginia, with funding from the US National Science Foundation, is collaborating with seven US science societies and the RAISE Project (www.raiseproject.org), which campaigns to raise the status of professional women through better recognition of their achievements.

We urge societies to use gender-neutral descriptors to broaden their candidate pool. Committees should establish selection criteria before reviewing nominees, and committee members need to understand the impact of implicit bias.

Anne E. Lincoln Southern Methodist University, Texas, USA. Stephanie H. Pincus The RAISE Project, USA.

Phoebe S. Leboy Association for Women in Science, USA; University of Pennsylvania, USA. phoebe@biochem.dental.upenn. $e d u$

\section{Pitfalls of coal peak prediction}

Richard Heinberg and David Fridley argue that coal reserves may be exhausted within decades (Nature 468, 367-369; 2010), basing much of their analysis on fits of cumulative coal production to logistic functions in the style of M. King Hubbert, who famously predicted peak oil supply. But this method is problematic for example, fitting the decline in production of LP records to a logistic curve would incorrectly indicate that vinyl is a limited resource.

If scarcity were an important determinant of US coalproduction history, prices should have increased. Yet they have stayed around US\$34 per tonne for the past 50 years, irrespective of production trends. Alternative explanations could include changes in electricity demand and market structure. There would then be no justification for calculating the limit of coal resources from a logistic graph of production history.

The logistic fits that drive forecasts of coal exhaustion depend on which years are included in the analysis. Logistic fits using data up to 1989,1999 or 2009 forecast an ultimate coal reserve of 52,71 or 96 gigatonnes, respectively, and predict that production should have peaked in 1951, 1967 or 1986. In fact, coal production has increased since 1986 highlighting the weakness of the scarcity-driven Hubbert model in explaining production.

An exponential fit explains as much of the variation in US production data as does a logistic fit. Yet the interpretation of the two models is different: the logistic model predicts the end of coal; the exponential fit predicts an infinite supply. Supply is obviously not infinite, but without a theoretical framework to support the choice of a logistic fit, its prediction may be just as wrong.

The end of easy oil is driving a shift towards carbon-intensive options, such as oil-sands mining or converting coal to liquid fuel. We must rely on policy changes to ensure a less carbon-intensive future, not the end of cheap coal.

David Keith, Juan MorenoCruz University of Calgary, Alberta, Canada. keith@ucalgary.ca

\section{Treat ghostwriting as misconduct}

Research-funding agencies such as the US National Institutes of Health (NIH) still have no policies in place to prevent either ghostwriting or guestwriting, despite the importance of authorship in career advancement (Nature 468,732 ; 2010).

Ghostwriting is the unacknowledged authoring of scientific papers by industry, and guestwriting includes authors who have made little contribution to the work: both affront academic principles.

Cases involving NIH-funded researchers might, the agency confusingly suggests, be regarded as plagiarism and so could be subject to federal regulations on research (go.nature.com/i5bnzo). The University of Pennsylvania School of Medicine's authorship policies also equate ghostwriting with plagiarism. However, neither ghost nor guest plagiarize anyone, and the putative 'victim' - the company - simply invites researchers to publish work under their own names (T. D. Anekwe Bioethics 24, 267-272; 2010).

The 2009 Danish law on scientific dishonesty regards misappropriation of authorship as research misconduct, defining it as 'false credit given to the author or authors, misrepresentation of title or workplace' (see go.nature. com/rj9slh and go.nature.com/ kqzgsc).

The US Office for Research Integrity and others, such as the UK Research Integrity Office and the European Network of Research Integrity Offices, should follow the Danish example by introducing and enforcing regulatory policies on ghost- and guestwriting. Like fabrication and falsification, guest- and ghostwriting should be treated as research misconduct, not plagiarism. Xavier Bosch Department of Internal Medicine, Hospital Clinic, University of Barcelona, Spain. xavbosch@clinic.ub.es

\section{CORRECTION}

In Poonam Kaushik Bakhshi's Correspondence (Nature 469, 162; 2011), we mistakenly attributed the author's proposed fern bioremediation strategy to the Hungarian government. 